\title{
Perfis das estudantes dos cursos de Informática do Instituto Federal de Educação, Ciência e Tecnologia de São Paulo (IFSP), campus Bragança Paulista
}

\author{
Thais Bergens de Sousa ${ }^{1}$, Elisandra Aparecida Alves da Silva ${ }^{\mathbf{1}}$, Sandra Cristina \\ Martins de Oliveira ${ }^{1}$ \\ ${ }^{1}$ Instituto Federal de Educação, Ciência e Tecnologia de São Paulo (IFSP), campus \\ Bragança Paulista \\ Av. Major Fernando Valle, 2013 - 12903-000 - Bragança Paulista, SP - Brasil \\ thaisbergens2000@gmail.com, \{elisandra, sandra.martins\}@ifsp.edu.br
}

\begin{abstract}
It is important to assess the students profile at the Information Technology (IT) courses in the IFSP - Bragança Paulista, to collaborate with the inclusion and permanence of the students in the field and to promote the participation of women in the job market as well. The campus provides technical courses integrated to the High School, Higher Education and Post-Graduation in IT, being possible to act in different educational levels. This work relates to an extension project that aims to promote the participation of women in Exact Sciences. Therefore, the purpose of this paper is to bring forward the profile of students in different school levels at the IT field in the campus and, based on the results, define proper strategies.
\end{abstract}

Resumo. É importante compreender os perfis das estudantes dos cursos de Informática do IFSP - Bragança Paulista, para colaborar na inclusão e na permanência das estudantes na área e, também, na promoção da participação de mulheres no mercado de trabalho. O campus possui curso técnico integrado ao ensino médio, superior e pós-graduação na área de Informática, sendo possivvel atuar nos diferentes níveis de ensino. Ademais, este trabalho está relacionado a um projeto de extensão que busca promover a participação de mulheres na área de Exatas. Dessa forma, o objetivo deste artigo é apresentar os perfis das estudantes dos diferentes níveis de ensino na área Informática do campus e com esses resultados definir estratégias adequadas.

\section{Introdução}

Segundo o Unlocking the Power of Women In Technology (UPWIT) [UPWIT 2018, p. 8], no Brasil, a área de tecnologia não é tão inovadora quanto deveria, pois apresenta desigualdades de gênero e etnia desde o acesso até oportunidades e salários.

Os cursos de computação possuem um alto número de estudantes e egressos e de acordo com as informações divulgadas em 2014 pela Sociedade Brasileira de Computação (SBC) [SBC 2014], formaram-se 39.342 profissionais nesta área no país, sendo que deste total apenas $16 \%$ são mulheres. 
Segundo o Relatório de Monitoramento Global da Educação - resumo 2017/8 [Unesco 2017, p. 44], o mundo alcançou a meta de paridade de gênero em todos os níveis acadêmicos, exceto na educação terciária. As disparidades de gênero nos resultados do ensino superior apresentam, muitas vezes, padrões entre os diferentes campos de estudo.

Em várias áreas do conhecimento, como mostra o relatório Gender in the Global Research Landscape [Elsevier 2017, p. 22], as mulheres representam, pelo menos 35\% dos profissionais na maioria dos países analisados. No entanto, em Energia, Engenharia, Matemática, Física, Astronomia e, principalmente, em Ciência da Computação, as mulheres representam menos de $25 \%$ dos profissionais na maioria dos países considerados na pesquisa.

Ademais, conforme a Pesquisa Nacional de Amostra de Domicílios do IBGE 2009, no mercado de trabalho apenas $20 \%$ dos 580 mil profissionais da área de Tecnologia da Informação do Brasil são mulheres [PNAD 2009]. Além disso, o UPWIT (2018, p.8), aponta que o salário dessas mulheres em 2010 era $34 \%$ menor em relação ao salário dos homens.

Percebe-se, dessa forma, que a participação de mulheres nas Ciências, Tecnologias, Engenharias e Matemática ainda é muito pequena e promover a inclusão das mulheres respeitando a diversidade de gênero é muito importante para que isso mude.

O programa Meninas Digitais da Sociedade Brasileira de Computação (SBC) visa estimular a participação de mulheres na área de Computação e das Tecnologia da Informação e Comunicação. Sendo voltado para alunas do ensino médio e dos últimos anos do ensino fundamental, o programa realiza diversas ações, como fóruns, palestras e oficinas e conta com projetos parceiros executados em diversas regiões do Brasil [Bim, Figueiredo, Maciel 2018].

A ONU determinou a Agenda 2030 para o Desenvolvimento Sustentável, que é um plano de ação para as pessoas, para o planeta e para a prosperidade [PNUD 2015]. A Agenda 2030 apresenta 17 objetivos de Desenvolvimento Sustentável e 169 metas que buscam concretizar os direitos humanos e alcançar a igualdade de gênero e o empoderamento das mulheres e meninas. Os objetivos são integrados e equilibram as três dimensões do desenvolvimento sustentável: a econômica, a social e a ambiental [ODM Brasil 2018].

O objetivo desse trabalho é determinar o perfil das estudantes dos cursos da área de Informática do Instituto Federal de Educação, Ciência e Tecnologia de São Paulo, campus Bragança Paulista (IFSP-BRA) e com os resultados elaborar estratégias eficientes para os cursos e projeto de extensão $\operatorname{IF}$ (meninas)\{nas exatas\}, no qual este trabalho está inserido. Como objetivos específicos, pretende-se verificar como é a participação das mulheres nos três níveis de formação oferecidos no IFSP-BRA.

\section{Metodologia}

Para determinação dos perfis das estudantes dos cursos de Informática, a metodologia definida engloba duas etapas:

(1) Avaliação quantitativa a partir de dados extraídos do Sistema Nacional de Informações da Educação Profissional e Tecnológica (SISTEC) e do Sistema 
Unificado de Administração Pública (SUAP), tratados com a estatística descritiva no Excel e estatística gráfica gerada no software Tableau. Os cursos considerados foram o Técnico Integrado em Informática, Tecnologia em Análise e Desenvolvimento de Sistemas e Pós-graduação em Gestão Estratégica de Tecnologia da Informação, pois são os cursos da área de Informática oferecidos atualmente no campus. O período de análise foi o de criação de cada curso até o ano atual;

(2) Avaliação qualitativa, e partir da elaboração de questionários, utilizando o Formulário Google, os quais foram submetidos ao Comitê de Ética em Pesquisa, aprovados e respondidos pelos estudantes matriculados. Ademais, foram atribuídos questionários para os diferentes cursos (técnico, graduação e pósgraduação), visto que existem questões específicas para cada nível de ensino. A seguir, apresenta-se os resultados obtidos.

\section{Resultados e discussões}

O período observado na avaliação quantitativa foi desde a criação de cada curso até o ano de 2019. Os anos ou semestres mostram o início do ciclo escolar de cada turma.

O curso Técnico Integrado em Informática, implantado em 2012, oferece 40 vagas anualmente no período integral e tem duração de 3 anos. Na Figura 1, o gráfico (1) mostra que a presença de mulheres é muito boa no campus, sendo que nos últimos dois anos o ingresso de mulheres foi muito expressivo e observando a etnia, a maioria das ingressantes é branca ou não declarada, sendo que a participação de meninas pretas é muito baixa.

No curso de Tecnologia em Análise e Desenvolvimento de Sistemas, gráfico (2) da Figura 1, implantado em 2009, são oferecidas 40 vagas semestralmente no período noturno e possui duração de 3 anos. Os dados apresentados evidenciam que a participação de mulheres nesse nível é muito inferior à de homens. No ano de 2013 houve a migração de estudantes para a nova grade, por esse motivo, há um número maior de ingressantes. Em relação a etnia e considerando as informações declaradas a participação maior é de mulheres brancas e há poucas mulheres pretas no nível superior.

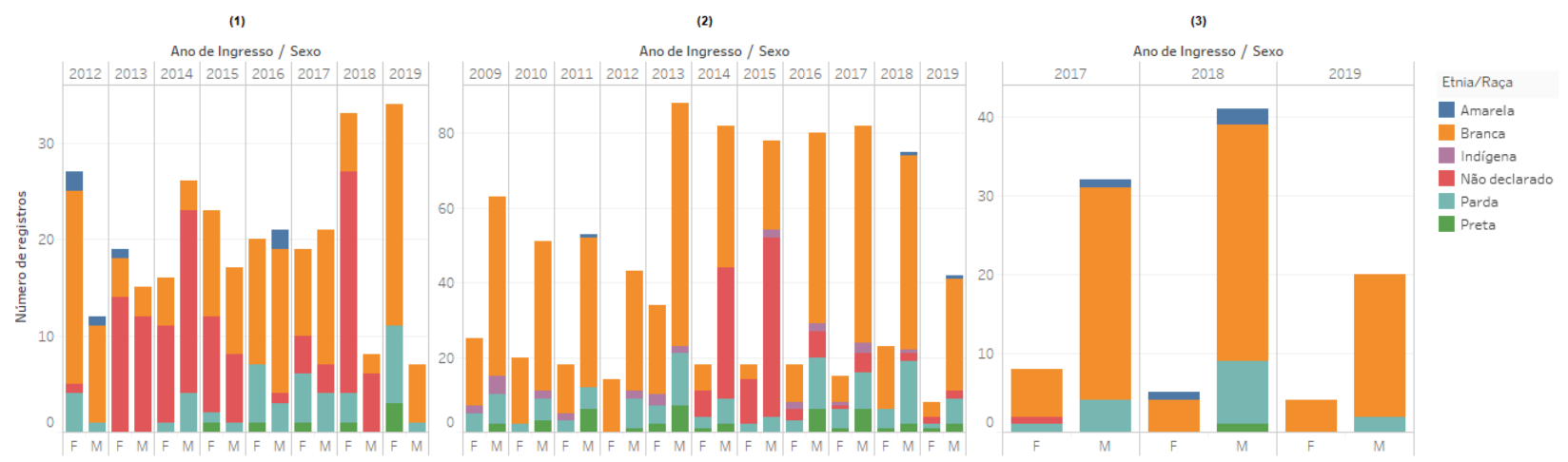

Figura 1. Ingressantes dos cursos da área de Informática 
O curso de Pós-graduação em Gestão Estratégica de Tecnologia da Informação, implantado em 2017, oferece 20 vagas semestralmente no período noturno e tem duração de 1 ano e meio. Os dados apresentados no gráfico (3) da Figura 1 confirmam a baixa participação de mulheres (menos de 16\%), sendo na sua maioria branca, com nenhuma participação de mulheres pretas.

Focando na análise qualitativa, os três questionários elaborados (técnico, graduação e pós-graduação) ${ }^{1}$ englobam perguntas comuns. Neste artigo, são apresentadas as respostas para a pergunta: "Quais as principais dificuldades no curso?".

No curso Técnico Integrado em Informática, 57 estudantes responderam o questionário, sendo que desse total 40 são meninas. As principais dificuldades apontadas pelas estudantes no nível médio são apresentadas na nuvem de palavras (1) apresentada na Figura 2, o que demonstra dificuldades na área técnica, além da falta de tempo, que é a dificuldade principal apontada.

(1)

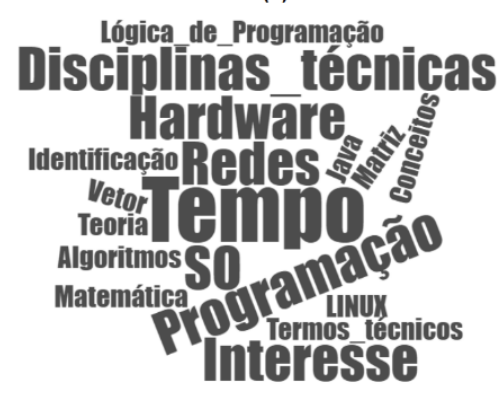

(2)

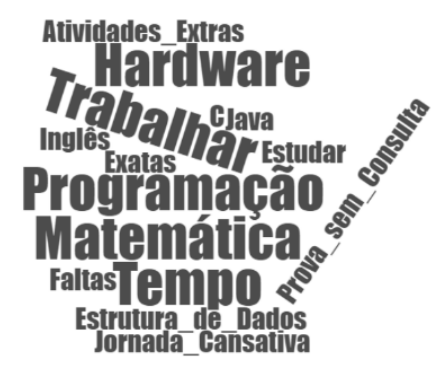

(3)

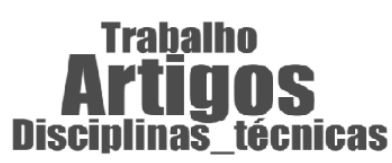

\section{Figura 2. Dificuldades das estudantes nos cursos do IFSP-BRA}

No curso de Tecnologia em Análise e Desenvolvimento de Sistemas, 105 estudantes responderam o questionário, sendo 26 do sexo feminino. As principais dificuldades das estudantes no nível superior, apresentadas na nuvem (2) da Figura 2 estão relacionadas a programação, matemática, conciliar trabalho e estudos e a falta de tempo.

No curso da pós-graduação em Gestão Estratégica de Tecnologia da Informação, 49 alunos responderam o questionário e apenas 7 são mulheres. As principais dificuldades das estudantes na pós-graduação são a elaboração de artigos e as disciplinas técnicas.

\section{Conclusões}

Os resultados da análise quantitativa indicam um aumento considerável no ingresso de meninas nos dois últimos anos, que é maior do que $80 \%$. Uma das mudanças ocorridas nesses últimos anos foi a forma de ingresso, que foi alterada de prova para análise do histórico escolar (notas de Português e Matemática) do $8^{\circ}$ ano do ensino fundamental, mas é necessário um estudo mais amplo para compreender os motivos desse crescimento.

Os dados do curso superior e pós-graduação indicam uma baixa participação de mulheres, sendo muito baixa a de mulheres pretas na graduação e nula na pós-graduação, o que nos mostra que precisamos definir estratégias para as mulheres pretas conhecerem

1

Disponíveis em: http://bra.ifsp.edu.br/ifmeninas/ 
os cursos e ingressarem na área, por meio de visitas a escolas públicas de nível médio e de graduação da região e elaboração de oficinas técnicas direcionadas para esse público.

Percebe-se pelos resultados apresentados que as principais dificuldades das meninas no nível médio são a falta de tempo, redes e programação. Dessa forma, é necessário desenvolver atividades para melhorar a organização do tempo, considerando a criação de grupos de estudos, oficinas e clubes de programação.

Na graduação, observa-se uma pequena participação de mulheres. Além disso, as principais dificuldades envolvem programação, matemática e falta de tempo. É importante também neste nível oferecer mecanismos para o desenvolvimento de projetos mais práticos, relacionados ao mercado de trabalho, grupos de estudos e oficinas técnicas.

Na pós-graduação as principais dificuldades estão na elaboração de artigos e disciplinas técnicas, o que nos orienta na definição de trabalhos mais práticos.

As análises realizadas são um passo importante para traçar os perfis das estudantes nos diferentes níveis e tendo em vista que o IFSP-BRA oferece cursos nos 3 níveis de formação, pode-se atuar fortemente para manter as estudantes na área.

\section{Referências}

Bim, S. A.; Figueiredo, K. S.; Maciel, C. (2018). Digital Girls Program-Disseminating Computer Science to Girls in Brazil. In: 40th International Conference on Software Engineering, GE@ICSE018, Gothenburg, Sweden.

Elsevier. (2018). Gender in the Global Research Landscape. Disponível em: $<$ https://www.elsevier.com/_data/assets/pdf_file/0008/265661/ElsevierGenderRepo rt_final_for-web.pdf $>$. Acesso em: 25 maio 2018 .

ODM Brasil. (2018). Os Objetivos de Desenvolvimento do Milênio. Disponível em: $<$ http://www.odmbrasil.gov.br/os-objetivos-de-desenvolvimento-do-milenio $>$. Acesso em: 10 abr. 2018.

PNAD. (2009). Pesquisa Nacional por Amostra de Domicílios do IBGE 2009. Disponível em: < https://biblioteca.ibge.gov.br/>. Acesso em: 15 abr. 2018.

PNUD. (2018). Os Objetivos de Desenvolvimento Sustentável: dos ODM aos ODS. Disponível em: <http://www.br.undp.org/content/brazil/pt/home/post-2015.html $>$. Acesso em: 20 abr. 2018.

SBC. (2014). Sociedade Brasileira de Computação. Disponível em: $<$ http://www.sbc.org.br>. Acesso em: 10 abr. 2018.

Unesco. (2018). Relatório de Monitoramento Global da Educação - resumo 2017/8: responsabilização na educação: cumprir nossos compromissos. Disponível em: $<$ http://unesdoc.unesco.org/images/0025/002595/259593por.pdf >. Acesso em: 18 abr. 2018.

UPWIT. (2018). Mulheres líderes na tecnologia: como promover a equidade de gênero e reter talentos nas empresas. Disponível em: <http://upwit.org/E-book-MulheresLideres-na-Tecnologia-1>. Acesso em: 22 mar. 2018. 